\title{
INVARIANT SUBGROUPS OF GROUPS OF HIGHER DERIVATIONS
}

\author{
JAMES K. DEVENEY AND JOHN N. MORDESON
}

\begin{abstract}
Let $L$ be a field of characteristic $p>3$. A subgroup $G$ of the group $D$ of all rank $p^{e}$ higher derivations on $L$ is $G$ alois if $G$ is the group of all $d$ in $D$ having a given subfield in its field of constants. The field of constants of $G$ is denoted as $L^{G}$. The main result states: Let $H \subseteq G$ be Galois subgroups of $D$. Then $H$ is an invariant subgroup of $G$ if and only if either $L^{H}=L^{G}\left(L^{p^{\prime}}\right)$ for some nonnegative integer $r$, or $L^{H} \subseteq L^{G}\left(L^{p^{q}}\right)$.
\end{abstract}

Let $L$ be a field of characteristic $p \neq 0$. A rank $p^{e}$ higher derivation on $L$ is a sequence of additive maps $d=\left\{d_{0}, d_{1}, \ldots, d_{p^{e}}\right\}$ such that $d_{r}(a b)=$ $\Sigma\left\{d_{i}(a) d_{j}(b) \mid i+j=r\right\}$ and $d_{0}$ is the identity map. Let $D$ be the group of all rank $p^{e}$ higher derivations on $L$ where the group operation is defined by $d \circ e=f$ where $f_{r}=\Sigma\left\{d_{i} e_{j} \mid i+j=r\right\}$. For $G$ a subset of $D$, its field of constants is $\left\{a \in L \mid d_{r}(a)=0, r>0, d \in G\right\}$ and will be denoted by $L^{G}$. A subgroup $G$ of $D$ is Galois if it is the group of all higher derivations which are trivial on $L^{G}$. For $F$ an intermediate field of $L / L^{G}$, we let $D^{F}$ denote the group of all those $d \in D$ whose field of constants contains $F$. For $H$ and $G$ subgroups, $H$ is $G$-invariant if $d^{-1} d^{\prime} d \in H$ for all $d^{\prime} \in H, d \in G$. The following is the main result.

TheOREM. Suppose $p>3$. Let $H \subseteq G$ be Galois subgroups of $D$. Then $H$ is $G$-invariant if and only if either $L^{H}=L^{G}\left(L^{p^{\prime}}\right)$ for some nonnegative integer $r$, or $L^{H} \subseteq L^{G}\left(L^{p^{e}}\right)$.

This result can be immediately applied to [3, Theorem 1, p. 338] to give a necessary and sufficient condition concerning group invariance for the inseparable Galois theory developed by Heerema [2]. For the purely inseparable finite dimensional case, [1, Theorem (3.1), p. 289] shows that $H$ being $G$-invariant is not equivalent to $L^{H}$ being invariant under elements of $G$, unless $L$ is of exponent less than $e+1$ over $L^{G}$. However, even in this case when $G / H$ acts as a group of higher derivations on $L^{H}$, it will in general not be a Galois group [1, Theorem (3.4), p. 289], although the field of constants of the restrictions to $L^{H}$ of $G$ will be $L^{G}[1,2.5(\mathrm{c})$, p. 289]. It seems interesting that in the case where $L / L^{G}$ is of exponent less than $e+1, G$ will have only

Received by the editors April 19, 1977 and, in revised form, August 29, 1977.

AMS (MOS) subject classifications (1970). Primary 12F15.

Key words and phrases. Purely inseparable field extension, higher derivation, Galois group of higher derivations. 
a finite number of normal Galois subgroups. We note that $L / L^{G}$ is modular and hence has a subbasis $\left[4\right.$, Theorem 1, p. 403] since $L / L^{G}$ is of exponent less than or equal to $e+1$. We frequently use the fact that a higher derivation on $L$ is uniquely determined by its action on a $p$-basis for $L$ [1, (2.1), p. 264]. We also assume $e>0$, since in the case $e=0, D$ is commutative and the Theorem is obvious.

Lemma. Let $H \subseteq G$ be Galois subgroups of $D$. Suppose there exist $m_{1}, m_{2}$ in $L$ such that $m_{1}$ is in a subbasis $M_{1}$ of $L / L^{G}, m_{2}$ is in a subbasis $M_{2}$ of $L / L^{H}$, and $t_{1}<\min \left\{s_{1}, t_{2}, e\right\}$, where $t_{1}, s_{1}$ are the exponents of $m_{1}$ over $L^{H}, L^{G}$, respectively, and $t_{2}$ is the exponent of $m_{2}$ over $L^{H}$. Suppose $H$ is $G$-invariant. Then $p .=2$ or $p=3$.

Proof. Define $d=\left\{d_{0}, \ldots, d_{p^{e}}\right\}$ and $d^{\prime}=\left\{d_{0}^{\prime}, \ldots, d_{p^{\prime}}^{\prime}\right\}$ in $D$ as follows:

$d_{i}(b)=0, i=1, \ldots, p^{e}$, for all $b \in M_{1}-\left\{m_{1}\right\}$,

$d_{i}\left(m_{1}\right)=0, i=1, \ldots, q-1, d_{q}\left(m_{1}\right)=m_{2}$,

$d_{i}^{\prime}(b)=0, i=1, \ldots, p^{e}$, for all $b \in M_{2}-\left\{m_{2}\right\}$,

$d_{i}^{\prime}\left(m_{2}\right)=0, i=1, \ldots, q^{\prime}-1, d_{q^{\prime}}^{\prime}\left(m_{2}\right) \neq 0$,

where $q=p^{e-s_{1}}+1$ if $s_{1}<e+1$ and $q=1$ if $s_{1}=e+1, q^{\prime}=p^{e-t_{2}}+1$ if $t_{2}<e+1$ and $q^{\prime}=1$ if $t_{2}=e+1$.

Suppose $t_{2}<e+1$. Then $d_{i}^{\prime}\left(m_{2}^{p^{t_{2}}}\right)=\left(d_{j}^{\prime}\left(m_{2}\right)\right)^{p^{t_{2}}}$ if $i=j p^{t_{2}}$ for some $j$ and $d_{i}^{\prime}\left(m_{2}^{p^{\prime 2}}\right)=0$ otherwise [6, p. 436]. Suppose $i=j p^{t_{2}}$ for some $j$. Then $j p^{t_{2}} \leqslant p^{e}$ so $j \leqslant p^{e-t_{2}}<q^{\prime}$. Thus $d_{i}^{\prime}\left(m_{2}^{p^{\prime 2}}\right)=0$. Hence $d^{\prime} \in H$. Similarly, $d \in G$ if $s_{1}<e+1$. If $t_{2}=e+1$ or $s_{1}=e+1$, then it follows easily that $d^{\prime} \in H$ and $d \in G$.

Now $p^{t_{1}}$ divides both $i$ and $\left(q+q^{\prime}\right) p^{t_{1}}-i$ if and only if $i=r p^{t_{1}}, r=$ $0,1, \ldots, q+q^{\prime}$. Assume $\left(q+q^{\prime}\right) p^{t_{1}} \leqslant p^{e}$. Then

$$
\begin{aligned}
& \sum\left\{d_{i}^{\prime} d_{\left(q+q^{\prime}\right) p^{t_{1}}-i}\left(m_{1}^{p^{t_{1}}}\right) \mid 0 \leqslant i \leqslant\left(q+q^{\prime}\right) p^{t_{1}}\right\} \\
& =\sum\left\{\left(d_{n}^{\prime} d_{q+q^{\prime}-n}\left(m_{1}\right)\right)^{p^{t_{1}}} \mid 0 \leqslant n \leqslant q+q^{\prime}\right\} \\
& =d_{\left(q+q^{\prime}\right) p^{t_{1}}}\left(m_{1}^{p_{1}^{t_{1}}}\right)+\left(d_{q^{\prime}}^{\prime} d_{q}\left(m_{1}\right)\right)^{p^{t_{1}}}+d^{\prime}\left(q+q^{\prime}\right) p^{t_{1}}\left(m_{1}^{p_{1}}\right) .
\end{aligned}
$$

Since

$$
d^{\prime}{ }_{\left(q+q^{\prime}\right) p^{t_{1}}}\left(m_{1}^{p_{1}^{t_{1}}}\right)=0 \text { and }\left(d_{q^{\prime}}^{\prime} d_{q}\left(m_{1}\right)\right)^{p^{t_{1}}} \neq 0
$$

we have that $d^{\prime} d \neq d$ on $L^{H}$, a contradiction. Thus $\left(q+q^{\prime}\right) p^{t_{1}}>p^{e}$. A routine solution of this inequality yields $p=2$ or $p=3$.

Proof of THE Theorem. Suppose $L^{H}=L^{G}\left(L^{p^{\prime}}\right)$. By [1, Theorem 1, p. 289], $L^{H}$ is invariant under $G$. Hence, clearly $H$ is $G$-invariant. Suppose $L^{H} \subseteq L^{G}\left(L^{p^{c}}\right)$. Since the pairs $L^{p^{c}}, L^{G}$ and $L^{p^{e}}, L^{H}$ are linearly disjoint, it follows that there exists a subset $B$ of $L$ such that $L^{H}=L^{G}\left(B^{p^{e}}\right)$. Let $d^{\prime}=\left\{d_{0}^{\prime}, \ldots, d_{p^{e}}^{\prime}\right\} \in H$ and $d=\left\{d_{0}, \ldots, d_{p^{e}}\right\} \in G$. For any $b \in B$ and $j=1, \ldots, p^{e}-1$,

$$
d_{j}\left(b^{p^{c}}\right)=0=\sum\left\{d_{i}^{\prime} d_{j-i}\left(b^{p^{e}}\right) \mid 0 \leqslant i \leqslant j\right\}
$$


since $p^{e}$ does not divide $j[6$, p. 436]. Now

$$
\sum\left\{d_{i}^{\prime} d_{p^{e}-i}\left(b^{p^{e}}\right) \mid 0 \leqslant i \leqslant p^{e}\right\}=d_{p^{e}}\left(b^{p^{e}}\right)+d_{p^{e}}^{\prime}\left(b^{p^{e}}\right)=d_{p^{e}}\left(b^{p^{e}}\right) .
$$

Thus $d^{\prime} d=d$ on $L^{H}$, so $H$ is $G$-invariant.

If $L=L^{H}$ or $L^{H}=L^{G}$, the result is immediate. Hence assume $L \supset L^{H} \supset$ $L^{G}$ (strict inclusion). Since $L$ is modular over $L^{H}$ and $L^{G}\left(L^{p^{g}}\right), L$ is modular over $L^{H} \cap L^{G}\left(L^{p^{q}}\right)$ [5, Theorem 1.1, p. 39]. Set

$$
G^{\prime}=D^{L^{H} \cap L^{G}\left(L^{p^{e}}\right)} \text {. }
$$

Then $G^{\prime}$ is a Galois subgroup of $D, L^{G^{\prime}}=L^{H} \cap L^{G}\left(L^{p^{q}}\right), H \subseteq G^{\prime} \subseteq G$, and $H$ is $G^{\prime}$-invariant. Suppose $L^{H} \underline{Z} L^{G}\left(L^{p^{q}}\right)$. Then $L^{G^{\prime}} \subset L^{H}$ and $L^{H} \underline{Z}$ $L^{G^{\prime}}\left(L^{p^{q}}\right)$. Let $s$ denote the exponent of $L / L^{G^{\prime}}$. Suppose for all $m_{2}$ in $L$ of exponent $s$ over $L^{G^{\prime}}$ that $m_{2}^{p^{s-1}} \notin L^{H}$. Then for such an $m_{2}, m_{2}$ has maximal exponent over $L^{H}$, so $m_{2}$ is in a subbasis of $L / L^{H}$. There exists a subbasis of $L / L^{G^{\prime}}$ which contains an element $m_{1}$ such that the exponent $t_{1}$ of $m_{1}$ over $L^{H}<$ the exponent $s_{1}$ of $m_{1}$ over $L^{G^{\prime}}$ [3, Lemma 3, p. 340]. Since every element of $L$ with exponent $s$ over $L^{G^{\prime}}$ also has exponent $s$ over $L^{H}$, we have $t_{1}<s_{1}<s=t_{2} \leqslant e+1$. Hence $t_{1}<e$. Thus by the Lemma, $H$ is not $G^{\prime}$-invariant, a contradiction. Hence there exists $m$ in $L$ of exponent $s$ over $L^{G^{\prime}}$ such that $m^{p^{s-1}} \in L^{H}$. Let $m_{1}$ be such an element. Then $m_{1}$ is in a subbasis of $L / L^{G^{\prime}}$ and the exponent $t_{1}$ of $m_{1}$ over $L^{H}<$ the exponent $s_{1}=s$ of $m_{1}$ over $L^{G^{\prime}}$. Now $t_{1} \leqslant e$ and if $t_{1}=e$, then

$$
m_{1}^{p_{1}} \in L^{H} \cap L^{G}\left(L^{p^{e}}\right)=L^{G^{\prime}},
$$

a contradiction. Thus $t_{1}<e$. Suppose there exists $m_{2}$ in $L$ of exponent $s$ over $L^{G^{\prime}}$ such that $m p^{s-1} \notin L^{H}$. Then $m_{2}$ is in a subbasis of $L / L^{H}$ and $t_{1}<s=$ $t_{2}$. Thus by the Lemma, $H$ is not $G^{\prime}$-invariant, a contradiction. Hence for all $m_{2} \in L$ of exponent $s$ over $L^{G^{\prime}}, m_{2}^{p^{s-1}} \in L^{H}$. Thus $L^{G^{\prime}}\left(L^{p^{s-1}}\right) \subseteq L^{H}$.

We now conclude the proof by induction on the exponent of $L / L^{G}$. If the exponent of $L / L^{G}$ is 0 , then the result is immediate. Suppose

$$
L^{H}=L^{G^{*}}\left(L^{p^{\prime}}\right) \quad \text { or } \quad L^{H} \subseteq L^{G^{*}}\left(L^{p^{e}}\right)
$$

whenever $H \subseteq G^{*}$ are Galois subgroups of $D$ such that $H$ is $G^{*}$-invariant and the exponent of $L / L^{G^{*}}<n(n \geqslant 1)$. Let $H \subseteq G$ be Galois subgroups of $D$ such that $H$ is $G$-invariant and $L / L^{G}$ has exponent $n$. If $L^{H} \subseteq L^{G}\left(L^{p^{e}}\right)$, we have the desired result. Suppose $L^{H} \underline{Z} L^{G}\left(L^{p^{e}}\right)$. Let $s$ denote the exponent of $L /\left(L^{H} \cap L^{G}\left(L^{p^{g}}\right)\right)$. Then by the preceding paragraph, $L^{G}\left(L^{p^{s-1}}\right) \subseteq$ $L^{H}$. Now $s-1<n$ and $L / L^{G}\left(L^{p^{s-1}}\right)$ is modular. Let $G^{*}$ be the Galois subgroup of $D$ with field of constants $L^{G}\left(L^{p^{s-1}}\right)$. Then $H$ is $G^{*}$-invariant, and by the induction hypotheses,

$$
L^{H}=L^{G}\left(L^{p^{s-1}}\right)\left(L^{p^{r}}\right) \text { for some } r,
$$

or

$$
L^{H} \subseteq L^{G}\left(L^{p^{s-1}}\right)\left(L^{p^{e}}\right) .
$$

Suppose $L^{H} \subseteq L^{G}\left(L^{p^{s-1}}\right)\left(L^{p^{e}}\right)$. Since $s-1<e+1$, we have 


$$
L^{H} \subseteq L^{G}\left(L^{p^{s-1}}\right), \text { so } L^{H}=L^{G}\left(L^{p^{s-1}}\right) .
$$

Suppose $L^{H}=L^{G}\left(L^{p^{s-1}}\right)\left(L^{p^{\prime}}\right)$. Then

$$
L^{H}=L^{G}\left(L^{p^{s-1}}\right) \text { or } L^{H}=L^{G}\left(L^{p^{r}}\right) .
$$

In the following example, we show that for small $p$ it is possible for $H$ to be $G$-invariant without $L^{H}=L^{G}\left(L^{p^{\prime}}\right)$ for some $r$ or $L^{H} \subseteq L^{G}\left(L^{p^{e}}\right)$.

EXAMPLE. Let $L=P(x, y)$ where $P$ is a perfect field of characteristic $p=2$ or 3 and $x, y$ are independent indeterminates over $P$. Let $G$ and $H$ be the Galois subgroups of $D$ (with $e=2$ ) corresponding to $P\left(x^{p^{2}}, y^{p^{2}}\right)$ and $P\left(x^{p}, y^{p^{2}}\right)$, respectively. Since $d_{1}=0$ for any $d=\left\{d_{0}, d_{1}, \ldots, d_{p^{e}}\right\} \in G$, an easy computation shows $d^{\prime} d=d$ on $L^{H}$ for all $d^{\prime} \in H$.

\section{REFERENCES}

1. J. Deveney, An intermediate theory for a purely inseparable Galois theory, Trans. Amer. Math. Soc. 198 (1974), 287-295.

2. N. Heerema, A Galois theory for inseparable field extensions, Trans. Amer. Math. Soc. 154 (1971), 193-200. MR 42 \#4527.

3. J. Mordeson, On a Galois theory for inseparable field extensions, Trans. Amer. Math. Soc. 214 (1975), 337-347. MR 52 \#5635.

4. M. Sweedler, Structure of inseparable extensions, Ann. of Math. (2) 87 (1968), 401-410; correction, ibid. (2) 89 (1969), 206-207. MR 36 \#6391; 38 \#4451.

5. W. Waterhouse, The structure of inseparable field extensions, Trans. Amer. Math. Soc. 211 (1975), 39-56. MR 52 \#359.

6. M. Weisfeld, Purely inseparable extensions and higher derivations, Trans. Amer. Math. Soc. 116 (1965), 435-449. MR 33 \# 122.

Department of Mathematical Sciences, Virginia Commonwealth University, RichMOND, VIRGINIA 23284

Department of Mathematics, Creighton University, Omaha, Nebraska 68131 